\title{
Multi-centre, multi-vendor 7 Tesla fMRI reproducibility of hand digit representation in the human somatosensory cortex
}

\author{
Ian D Driver ${ }^{1}$, Rosa M Sanchez Panchuelo ${ }^{2}$, Olivier Mougin ${ }^{2}$, Michael Asghar $^{2}$, James Kolasinski ${ }^{1}$, William T \\ Clarke $^{3}$, Catarina Rua ${ }^{4}$, Andrew T Morgan ${ }^{5}$, Adrian Carpenter ${ }^{4}$, Keith Muir ${ }^{5}$, David Porter ${ }^{5}$, Christopher T \\ Rodgers $^{4}$, Stuart Clare ${ }^{3}$, Richard G Wise ${ }^{1,6,7}$, Richard Bowtell ${ }^{2}$, and Susan T Francis ${ }^{2}$ \\ ${ }^{I}$ Cardiff University Brain Research Imaging Centre, School of Psychology, Cardiff University, Cardiff, United \\ Kingdom \\ ${ }^{2}$ Sir Peter Mansfield Imaging Centre, School of Physics and Astronomy, University of Nottingham, Nottingham, \\ United Kingdom \\ ${ }^{3}$ Wellcome Centre for Integrative Neuroimaging, FMRIB, Nuffield Department of Clinical Neurosciences, \\ University of Oxford, Oxford, United Kingdom \\ ${ }^{4}$ Wolfson Brain Imaging Centre, Department of Clinical Neurosciences, University of Cambridge, Cambridge, \\ United Kingdom \\ ${ }^{5}$ Imaging Centre of Excellence, University of Glasgow, Glasgow, United Kingdom \\ ${ }^{6}$ Department of Neuroscience, Imaging and Clinical Sciences, "G. D'Annunzio University" of Chieti-Pescara, \\ Chieti, Italy \\ ${ }^{7}$ Institute for Advanced Biomedical Technologies, "G. D’Annunzio University" of Chieti-Pescara, Chieti, Italy
}

\begin{abstract}
Whilst considerable progress has been made in using ultra-high field fMRI to study brain function at fine spatial resolution, methods are generally optimized at a single site and do not translate to studies where multiple sites are required for sufficient subject recruitment. With a recent increase in installations of human $7 \mathrm{~T}$ systems, there is now the opportunity to establish a framework for multi-site $7 \mathrm{~T}$ fMRI studies. However, an understanding of the inter-site variability of fMRI measurements is required for datasets to be combined across sites. To address this, we employ a hand digit localization task and compare across-site and within-site reproducibility of $7 \mathrm{~T}$ fMRI to a hand digit localization task which requires fine spatial resolution to resolve individual digit representations. As part of the UK7T Network "Travelling Heads" study, 10 participants repeated the same hand digit localization task at five sites with whole-body 7T MRI systems to provide a measure of inter-site variability. A subset of the participants ( 2 per site) performed repeated sessions at each site for measurement of intra-site reproducibility. Dice's overlap coefficient was used to assess reproducibility, with hand region inter-site Dice $=0.70 \pm 0.04$ significantly lower than intrasite Dice $=0.76 \pm 0.06$, with similar trends for the individual digit maps. Although slightly lower than intra-site reproducibility, the inter-site reproducibility results are consistent with previous single site reproducibility measurements, providing evidence that multi-site $7 \mathrm{~T}$ fMRI studies are feasible. These results can be used to inform sample size calculations for future multi-site somatomotor mapping studies.
\end{abstract}




\section{Introduction}

Functional magnetic resonance imaging (fMRI) provides a unique, non-invasive tool for resolving spatial features of neuronal modulations in the human brain, this is widely used to test hypotheses across the whole field of neuroscience research (Logothetis, 2008; Rosen and Savoy, 2012). The fMRI variant that shows the highest sensitivity without use of an exogenous contrast agent is the blood oxygenation level dependant (BOLD) contrast (Ogawa et al., 1990, 1992; Kwong et al., 1992).

BOLD fMRI can be performed at ultra-high field, higher field strengths than used for the majority of clinical workload (1.5-3 T), with the main advantage being finer spatial resolution. Increased sensitivity is available at high field, which can be traded for smaller voxel volumes (Triantafyllou et al., 2005). Considerable progress has been made recently in using 7 Tesla fMRI to study brain function at fine spatial resolution (Van der Zwaag et al., 2013; Puckett et al., 2017; Benson et al., 2018; Huber et al., 2020), however these protocols have been designed and optimised at a single research site and do not translate to studies where multiple sites are required to recruit the target population.

There has been a large recent expansion of 7 Tesla MRI infrastructure, driven by the release of the first commercially available clinical (CE-marked) 7 Tesla MRI system (U. S. Food \& Drug Administration, 2017), opening up the possibility of wide translation of 7 Tesla to clinical applications. Further, recent work from the UK 7T Network (Clarke et al., 2020; Rua et al., 2020), German Ultra-high Field Imaging Network (Voelker et al., 2016) and European Ultrahigh-Field Imaging Network in Neurodegenerative Diseases (Düzel et al., 2019) have started to establish protocols for harmonising 7 Tesla MRI measurements across sites and scanner models with good reproducibility, providing the framework to disseminate techniques across sites.

In order to assess fMRI reproducibility across $7 \mathrm{~T}$ sites an experimental design that exploits the advantages of ultra-high field is desirable, such as high spatial resolution. The study reported here investigates reproducibility of hand-digit representation measurements using 7 T fMRI across five centres, with measurements from three different 7 Tesla whole-body MRI systems from two different vendors. As part of the UK 7T Network's "Travelling-Heads" study, 10 participants were scanned at 5 sites, including a hand-digit localization fMRI task (Sanchez-Panchuelo et al., 2010; Kolasinski et al., 2016). The dataset from a single subject has been released as part of the UK 7T harmonization paper (Clarke et al., 2020) and this work has been presented in abstract format (Driver et al., 2021; https://www.ismrm.org/ $21 \mathrm{~m} /$ ).

\section{Methods}

Ten healthy subjects ( $32 \pm 6$ years; 3 female; 1 left-handed) participated in the study. To assess inter-site repeatability, the same protocol was repeated at five sites, using three different 7 Tesla whole-body MRI systems, as detailed in Table 1. The same model of volume-transmit, 32-channel receive head coil (Nova Medical) was used at each site. Subjects were split into five pairs, with each pair returning to one site an additional four times (two subjects per site), to assess intra-site repeatability. 


\begin{tabular}{|c|c|c|c|c|}
\hline \# & Site & Vendor & $\begin{array}{l}\text { Scanner } \\
\text { Model }\end{array}$ & $\begin{array}{l}\text { Gradient } \\
\text { Performance }\end{array}$ \\
\hline 1 & $\begin{array}{l}\text { Wolfson Brain } \\
\text { Imaging Centre, } \\
\text { University of } \\
\text { Cambridge }\end{array}$ & Siemens & $\begin{array}{l}\text { Magnetom } \\
\text { Terra }\end{array}$ & $\begin{array}{l}80 \mathrm{mT} \mathrm{m}^{-1} \\
200 \mathrm{mT} \mathrm{m}^{-1} \mathrm{~ms}^{-1}\end{array}$ \\
\hline 2 & $\begin{array}{l}\text { Cardiff University } \\
\text { Brain Research } \\
\text { Imaging Centre, } \\
\text { Cardiff University }\end{array}$ & Siemens & $\begin{array}{l}\text { Magnetom } \\
7 \mathrm{~T}\end{array}$ & $\begin{array}{l}70 \mathrm{mT} \mathrm{m}^{-1} \\
200 \mathrm{mT} \mathrm{m}^{-1} \mathrm{~ms}^{-1}\end{array}$ \\
\hline 3 & $\begin{array}{l}\text { Imaging Centre of } \\
\text { Excellence, } \\
\text { University of } \\
\text { Glasgow }\end{array}$ & Siemens & $\begin{array}{l}\text { Magnetom } \\
\text { Terra }\end{array}$ & $\begin{array}{l}80 \mathrm{mT} \mathrm{m}^{-1} \\
200 \mathrm{mT} \mathrm{m}^{-1} \mathrm{~ms}^{-1}\end{array}$ \\
\hline 4 & $\begin{array}{l}\text { Sir Peter Mansfield } \\
\text { Imaging Centre, } \\
\text { University of } \\
\text { Nottingham }\end{array}$ & Philips & $\begin{array}{l}\text { Achieva } \\
7 \mathrm{~T}\end{array}$ & $\begin{array}{l}40 \mathrm{mT} \mathrm{m}^{-1} \\
200 \mathrm{mT} \mathrm{m}^{-1} \mathrm{~ms}^{-1}\end{array}$ \\
\hline 5 & $\begin{array}{l}\text { Wellcome Centre for } \\
\text { Integrative } \\
\text { Neuroimaging } \\
\text { (FMRIB), University } \\
\text { of Oxford }\end{array}$ & Siemens & $\begin{array}{l}\text { Magnetom } \\
7 \mathrm{~T}\end{array}$ & $\begin{array}{l}70 \mathrm{mT} \mathrm{m}^{-1} \\
200 \mathrm{mT} \mathrm{m}^{-1} \mathrm{~ms}^{-1}\end{array}$ \\
\hline
\end{tabular}

Table 1: MRI System specifications for each site

Two runs of a visually cued, travelling wave somatomotor task were performed with the dominant hand. This entailed a visually paced sequential $1 \mathrm{~Hz}$ button press of $8 \mathrm{~s}$ blocks of digit movement, cycling across digit blocks from index finger (D2) to little finger (D5) in a "forward" run and from D5 to D2 in a "reverse" run (32s per four digits), with 8 cycles per run (Figure 1).

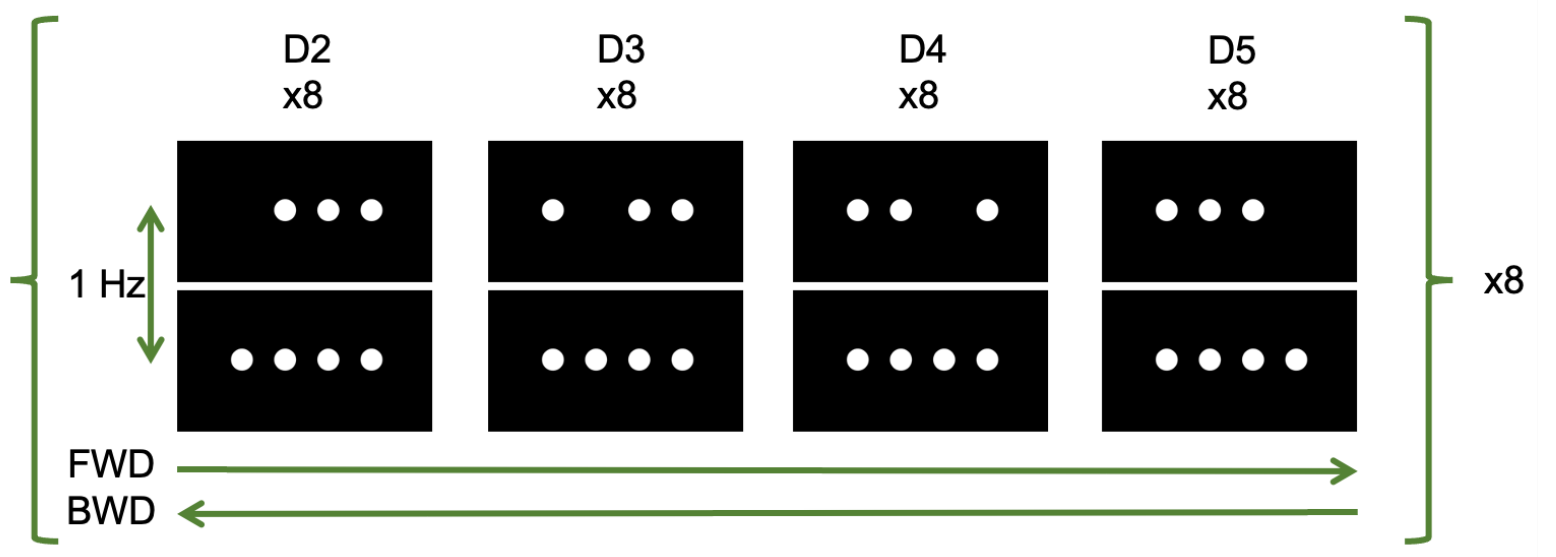

Figure 1: Hand digit localization task design. Participants were cued to press a button in time with a flashing circle, corresponding to each digit. FWD - "forward" run, from index finger (D2) to little finger (D4); BWD "reverse" run, from little finger to index finger. 
MRI calibration steps of radiofrequency transmit power calculation (using a 3DREAM $\mathrm{B}_{1}^{+}$ map) and $\mathrm{B}_{0}$ shimming were matched across sites, using the protocol that we introduced in our previous work (Clarke et al., 2020). Gradient-echo EPI protocol: 1.5mm isotropic resolution, $\mathrm{TR}=2 \mathrm{~s}, \mathrm{TE}=25 \mathrm{~ms}$, echo spacing 0.68/0.78ms Siemens/Philips, 34/28 slices Siemens/Philips. An in-plane acceleration factor of 2 was used, with GRAPPA for Siemens sites and SENSE for the Philips site. Spin-echo EPI scans were acquired with matched and reversed phaseencode direction for distortion correction (Driver et al., 2018; Clarke et al., 2020). A $\mathrm{T}_{1}$ weighted MPRAGE $\left(0.7 \mathrm{~mm}\right.$ isotropic, TR/TE/TI $\left.=2200 / 3.05 / 1050 \mathrm{~ms}, \mathrm{FA}=7^{\circ}\right)$ dataset was acquired for realignment and cortical flattening. $\mathrm{A} \mathrm{T}_{2}{ }^{*}$-weighted $2 \mathrm{D}$ FLASH $\left(0.75 \times 0.75 \times 1.5 \mathrm{~mm}^{3}, \mathrm{TE}=10 \mathrm{~ms}, \mathrm{TR}=1100 \mathrm{~ms}\right)$ dataset was acquired as an intermediate registration step between the EPI and $\mathrm{T}_{1}$-weighted MPRAGE.

Data was motion corrected using FSL MCFLIRT (Jenkinson et al., 2002), distortion corrected with FSL TOPUP (Andersson, Skare and Ashburner, 2003), and temporal filtered (100s cut-off) using FSL FEAT (Smith et al., 2004). A Fourier-based travelling wave analysis was performed using mrTools (Gardner et al., 2018) to calculate voxel-wise phase and coherence of the BOLD response. Image registration was performed using mrTools (Gardner et al., 2018). Whole-hand activation regions (FDR-corrected $\mathrm{p}<0.05$ ) were compared across sessions using Dice's overlap coefficient (Dice). An intersection mask was formed for each subject by including voxels identified as active across all five sites. Individual digit maps were formed within this intersection mask by dividing the phase maps into four equal $\pi / 2$ portions. Digit overlap across sessions was compared using the Dice similarity coefficient. Intra- and inter-site reproducibility were compared using Bonferronicorrected paired t-tests applied to the mean Dice coefficient across repeated sessions in the same site and across sites, respectively.

\section{Results}

Temporal SNR was similar across the five sites, with tSNR $=45 \pm 4 / 43 \pm 4 / 46 \pm 5 / 45 \pm 4 / 44 \pm 3$ (mean \pm standard deviation across subjects) for sites $1-5$, respectively and 1-way ANOVA $\mathrm{F}(4,45)=0.89, \mathrm{p}=0.48$, testing for differences in tSNR across sites. The comparison of tSNR was only considered across the 5 inter-site sessions, since including the repeat intra-site sessions would skew results to individual subjects, with only 2 subjects per site participating in the repeat sessions.

Figure 2 presents example conjunction maps for five subjects, showing the number of sessions with overlapping whole-hand activation regions, across repeats at the same site (intra-site), or across sites (inter-site). These datasets were chosen to present intra-site data from each of the five sites.

Phase (digit) maps are shown in Figure 3, with all sessions from a single subject shown (Fig. $3 a$ ) and all ten subjects' phase maps for a single site (Fig. 3b). The spatial distribution of the digit representations are consistent across sites for a single subject, whereas there is a high degree of inter-subject variability. Figure 4 shows both intra-site and inter-site conjunction maps for each digit, with a high degree of similarity between intra-site and inter-site for individual digits. 


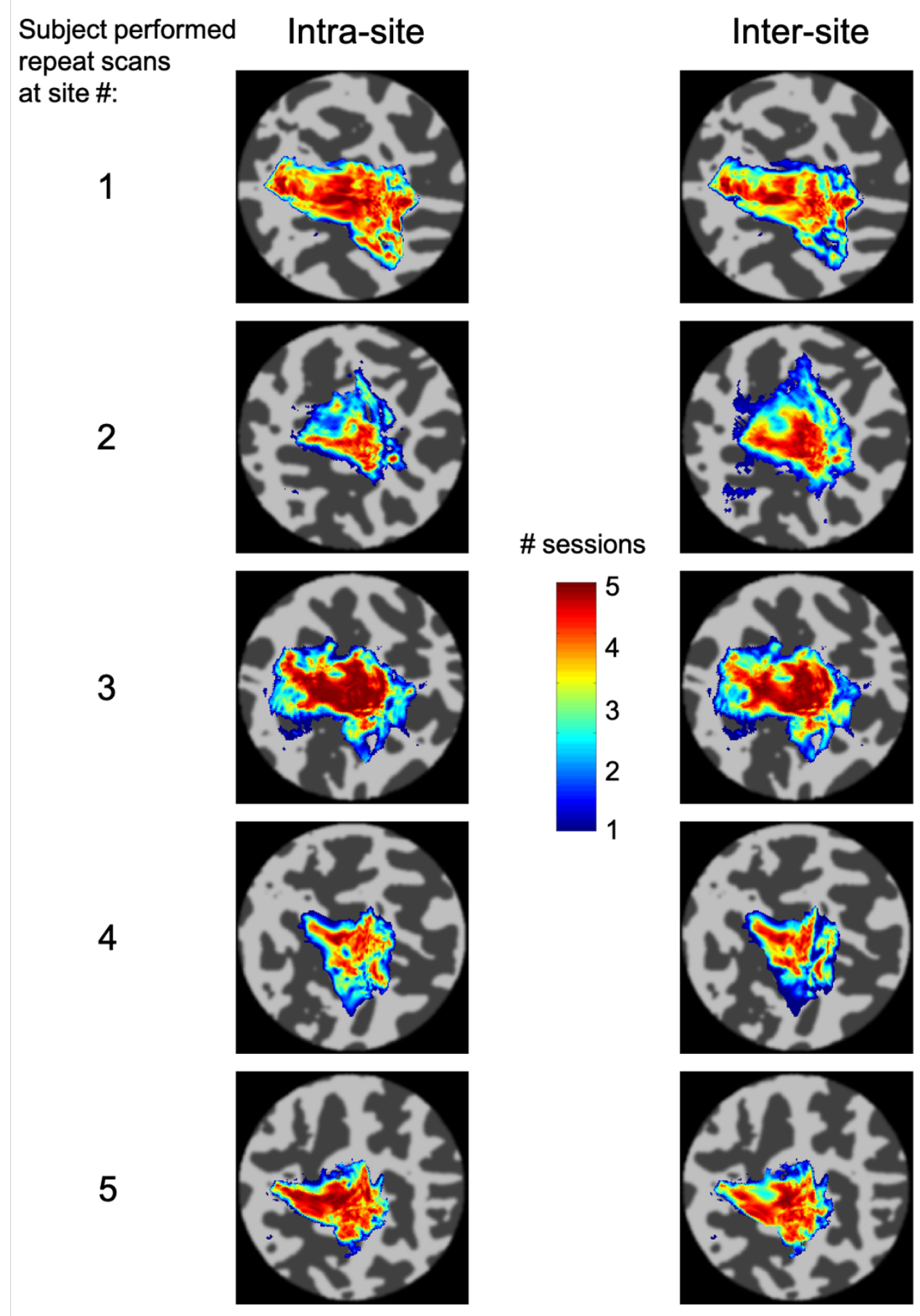

Figure 2: Conjunction maps for five subjects showing the overlap of the hand region between sessions for both repeat sessions for a single site (intra-site) or across sites (inter-site). The five subjects were chosen to represent intra-site measures for each site. 


\section{(a) Example subject dataset:}

4

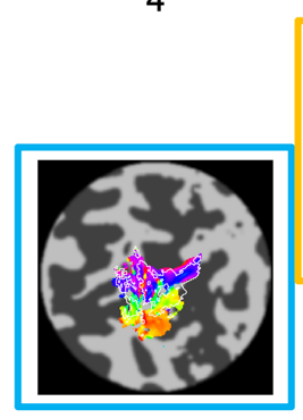

phase

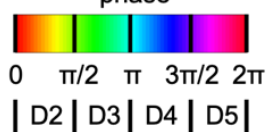

1
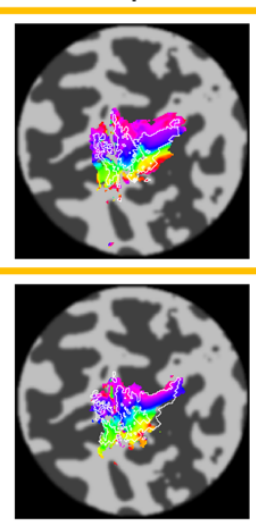

2
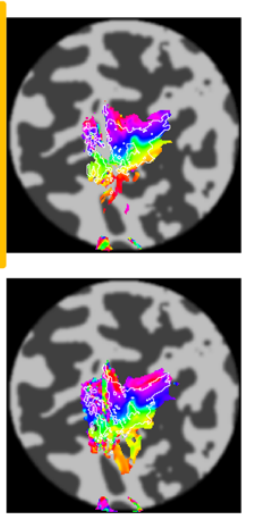

3
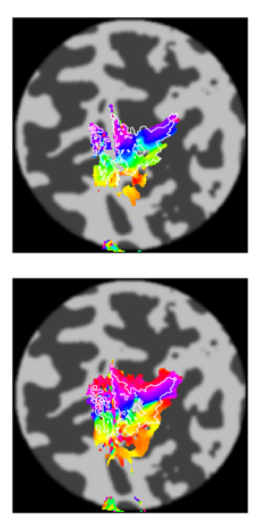

5
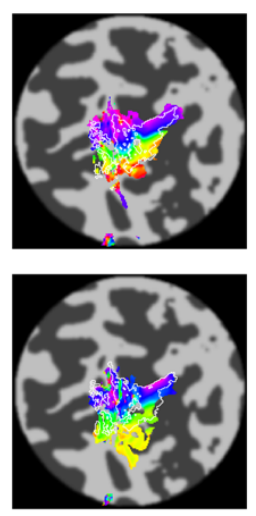

Site \#

Sites

Repeats at site 4

\section{(b) All subjects at the same site (site 1):}
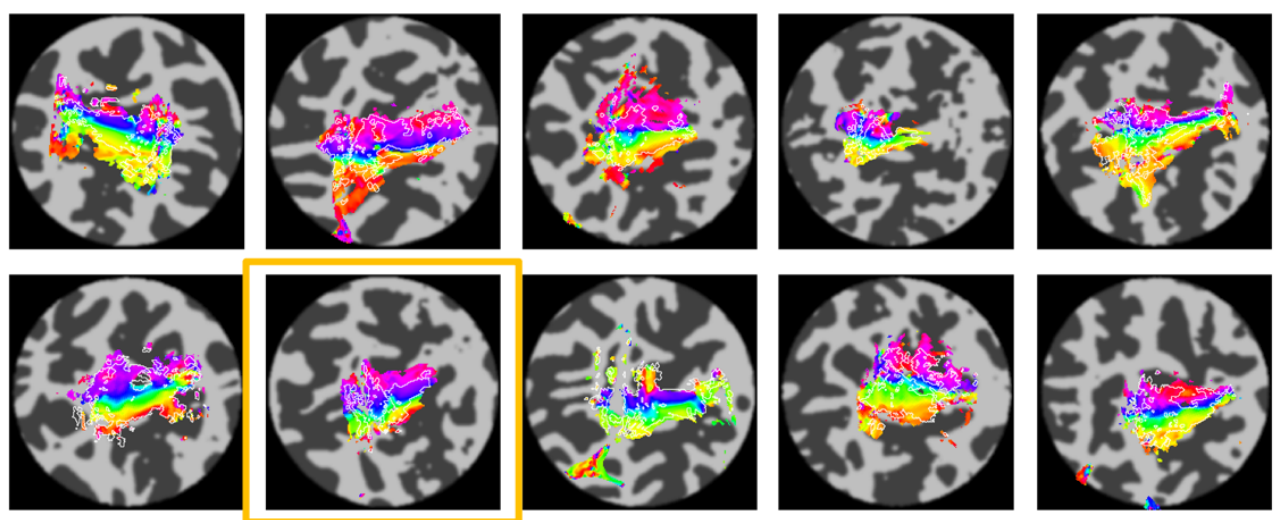

Figure 3: Phase maps and associated digit maps (D2-D5) defined by dividing phase into $\pi / 2$ portions. Overlay shown for $\mathrm{p}_{\mathrm{FDR}}<0.05$. Intersection mask outline shown in white ( $\mathrm{p}_{\mathrm{FDR}}<0.05$ hand region for all five sites). (a) Phase maps from all sessions for a single subject; (top row) all sites; (bottom row) repeat sessions at site 4. Blue border indicates data in both intra-site and inter-site analysis. (b) Phase maps from all 10 subjects for site 1. Orange border matches the session in (a). 
Dice coefficients for the whole hand region are higher for intra-session measures from the individual sites compared to inter-site measures (Figure $5 ; \mathrm{t}(9)=-5, \mathrm{p}_{\text {corr }}=0.002$ ). There is also a similar trend in the individual digit maps for intra-site Dice coefficients to be greater than inter-site (Fig. 5), but this only survives Bonferroni correction $\left(\mathrm{p}_{\text {corr }}<0.05\right)$ in D3 and D4.

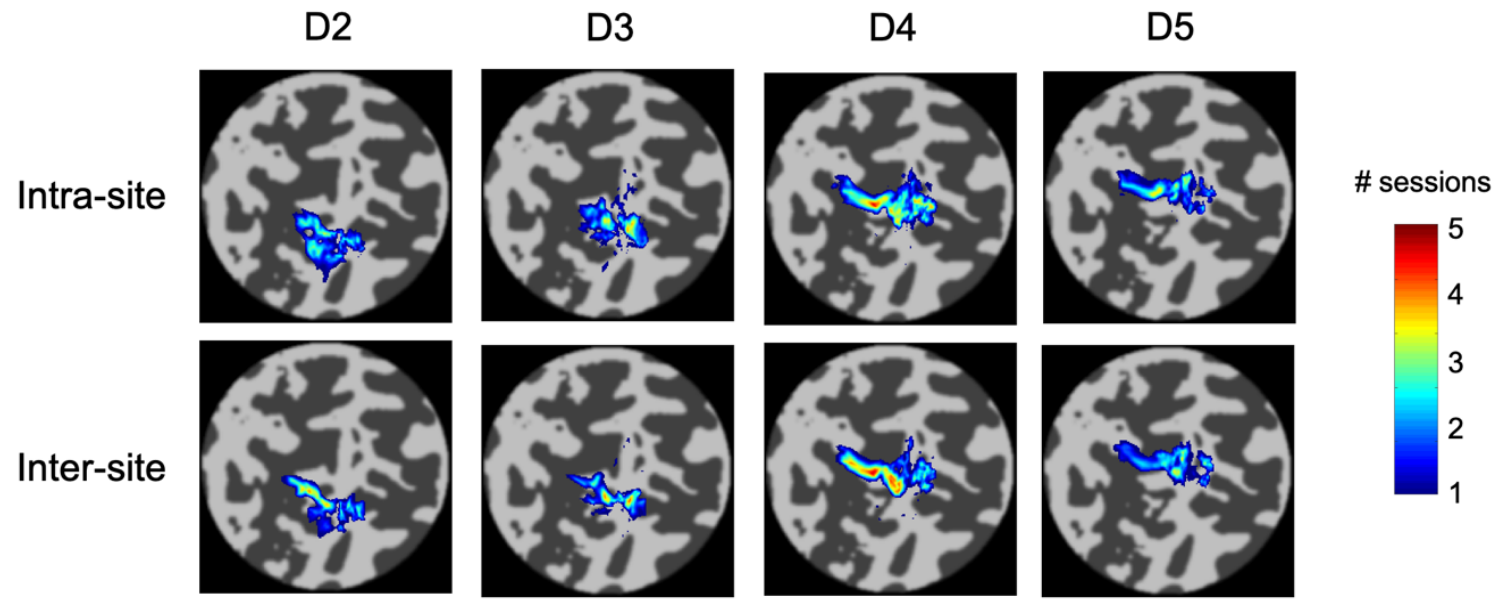

Figure 4: Conjunction maps for each digit (index finger - D2; little finger - D5) for a single subject, showing the overlap of the digit region between sessions for both repeat sessions for a single site (intra-site) or across sites (inter-site). The subject performed repeated sessions at site 4 .

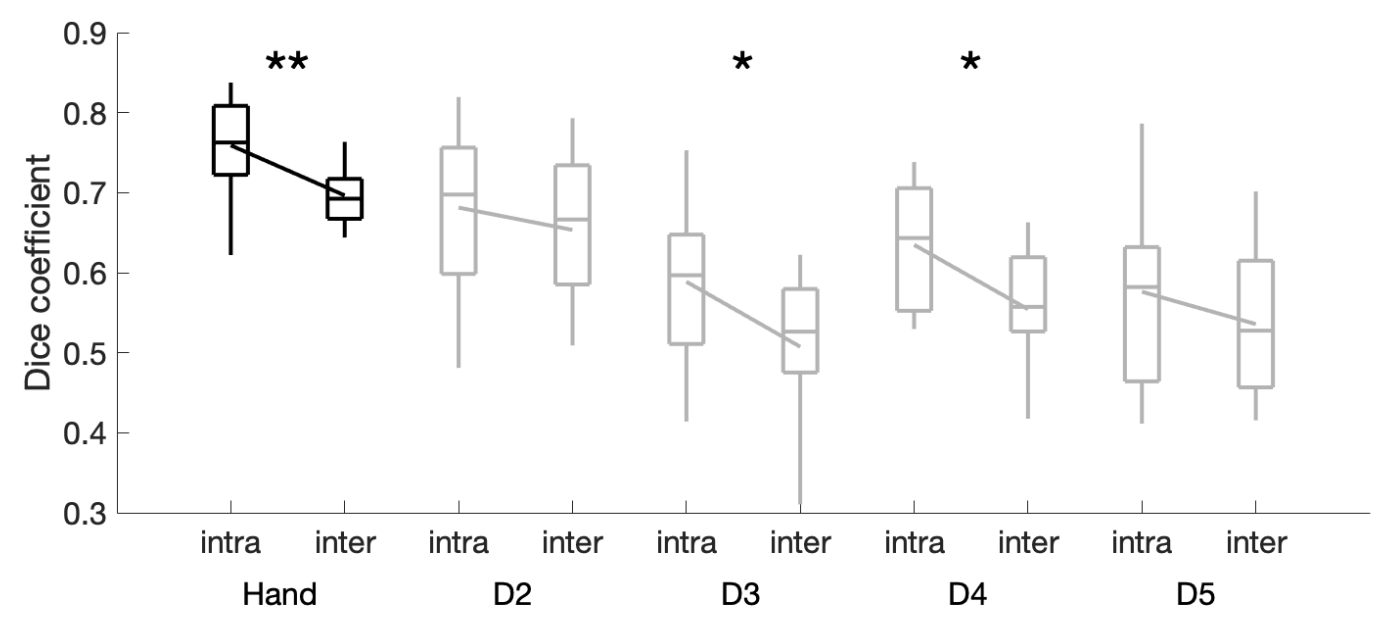

Figure 5: Boxplots comparing intra- and inter-site Dice coefficients for the whole hand region and each digit. Lines connecting intra/inter pairs compare their respective means. ${ }^{*} \mathrm{p}<0.005 ;{ }^{*} \mathrm{p}<0.05$ (Bonferroni-corrected) 


\section{Discussion}

This study demonstrates good reproducibility of fMRI digit maps across five, $7 \mathrm{~T}$ sites, using three different models of whole-body MRI system from two manufacturers. We did not harmonize the image reconstruction, coil combination, or parallel acquisition methods. Simple protocol harmonization, such as matching EPI echo train duration, in-plane acceleration factor, acquisition matrix, TE and TR, is sufficient to achieve good reproducibility across MRI systems, without needing to match gradient waveforms and radiofrequency pulse shapes.

The $1.5 \mathrm{~mm}$ isotropic spatial resolution fMRI data acquired here was able to resolve intersubject differences in hand digit representations, consistent with previous observations (Kolasinski et al., 2016). By repeating measurements across sites in the same participants, we were able to isolate inter-site variability from inter-subject differences. Qualitatively, inter-site and intra-site variability is small compared to these inter-subject differences. We show that the inter-site Dice overlap was smaller than intra-site. However, this difference in Dice coefficients corresponds to $\sim 5 \%$ difference in overlap in the regions between intra- and inter-site comparisons, which is small compared to the inter-session variability. Inter-site Dice values are similar to those reported across sessions in an intra-site study (Kolasinski et al., 2016). These findings can be used to power sample sizes required for future multi-site fMRI studies involving hand digit localization and show that such studies can gain from a larger participant pool available across multiple sites, with the increase in measurement variability from multisite measurements being small compared to the test-retest variability within a single site.

The region of interest definition was based on the coherence of the data studied, including voxels which were defined as active at all five sites. This approach risks missing novel features from individual sessions. Future work will define regions of interest based on a probabilistic atlas of hand digit areas (O'Neill et al., 2020). This approach will yield a broader region, including areas that may not be active in individual sessions, leading to increased variability in digit maps, however it will provide a region of interest definition that is independent of the data studied.

\section{Conclusion}

High resolution fMRI studies can be performed across multiple sites, with inter-site factors contributing. The inter-site variability measured here, in the form of Dice coefficients, can be used to inform future study designs and sample size calculations for multi-site somatomotor mapping studies.

\section{Acknowledgements and Funding}

The UK7T Network and this work was funded by the UK's Medical Research Council. [MR/N008537/1].

\section{Centre funding}

The Wellcome Centre for Integrative Neuroimaging is supported by core funding from the Wellcome Trust (203139/Z/16/Z).

Cardiff University Brain Research Imaging Centre is supported by the UK Medical Research Council (MR/M008932/1) and the Wellcome Trust (WT104943). 
This research was supported by the NIHR Cambridge Biomedical Research Centre (BRC1215-20014). The views expressed are those of the author(s) and not necessarily those of the NIHR or the Department of Health and Social Care. The Cambridge 7T MRI facility is cofunded by the University of Cambridge and the Medical Research Council (MR/M008983/1).

\section{Individual funding}

CTR is funded by a Sir Henry Dale Fellowship from the Wellcome Trust and the Royal Society [098436/Z/12/B].

\section{References}

Andersson, J. L. R., Skare, S. and Ashburner, J. (2003) 'How to correct susceptibility distortions in spin-echo echo-planar images: Application to diffusion tensor imaging', NeuroImage, 20(2). doi: 10.1016/S1053-8119(03)00336-7.

Benson, N. C. et al. (2018) 'The Human Connectome Project 7 Tesla retinotopy dataset: Description and population receptive field analysis', Journal of Vision. Association for Research in Vision and Ophthalmology Inc., 18(13), pp. 1-22. doi: 10.1167/18.13.23.

Clarke, W. T. et al. (2020) 'Multi-site harmonization of 7 tesla MRI neuroimaging protocols', NeuroImage, 206, p. 116335. doi: 10.1016/j.neuroimage.2019.116335.

Driver, I. D. et al. (2018) 'A comparison of distortion correction methods for EPI fMRI applied across three 7T platforms', in Proc. ISMRM, p. 5464.

Driver, I. D. et al. (2021) 'Multi-centre, multi-vendor 7 Tesla fMRI reproducibility of hand digit representation in the human somatosensory cortex', in Proc. ISMRM, p. 3372. Available at: https://www.ismrm.org/21m/.

Düzel, E. et al. (2019) 'European Ultrahigh-Field Imaging Network for Neurodegenerative Diseases (EUFIND)', Alzheimer's and Dementia: Diagnosis, Assessment and Disease Monitoring. Elsevier Inc, 11, pp. 538-549. doi: 10.1016/j.dadm.2019.04.010.

Gardner, J. L. et al. (2018) mrTools: Analysis and visualization package for functional magnetic resonance imaging data, Zenodo. doi: 10.5281/ZENODO.1299483.

Huber, L. et al. (2020) 'Sub-millimeter fMRI reveals multiple topographical digit representations that form action maps in human motor cortex', NeuroImage. Academic Press Inc., 208, p. 116463. doi: 10.1016/j.neuroimage.2019.116463.

Jenkinson, M. et al. (2002) 'Improved optimization for the robust and accurate linear registration and motion correction of brain images', NeuroImage, 17(2). doi: 10.1016/S10538119(02)91132-8.

Kolasinski, J. et al. (2016) 'Investigating the stability of fine-grain digit somatotopy in individual human participants', Journal of Neuroscience, 36(4), pp. 1113-1127. doi: 10.1523/JNEUROSCI.1742-15.2016.

Kwong, K. K. et al. (1992) 'Dynamic magnetic resonance imaging of human brain activity during primary sensory stimulation', Proceedings of the National Academy of Sciences of the United States of America. National Academy of Sciences, 89(12), pp. 5675-5679. doi: 10.1073/pnas.89.12.5675.

Logothetis, N. K. (2008) 'What we can do and what we cannot do with fMRI', Nature. 
Nature Publishing Group, pp. 869-878. doi: 10.1038/nature06976.

O’Neill, G. C. et al. (2020) 'A probabilistic atlas of finger dominance in the primary somatosensory cortex', NeuroImage. Elsevier Ltd, 217(May), p. 116880. doi: 10.1016/j.neuroimage.2020.116880.

Ogawa, S. et al. (1990) 'Oxygenation-sensitive contrast in magnetic resonance image of rodent brain at high magnetic fields', Magnetic Resonance in Medicine. John Wiley \& Sons, Ltd, 14(1), pp. 68-78. doi: 10.1002/mrm.1910140108.

Ogawa, S. et al. (1992) 'Intrinsic signal changes accompanying sensory stimulation: Functional brain mapping with magnetic resonance imaging', Proceedings of the National Academy of Sciences of the United States of America. National Academy of Sciences, 89(13), pp. 5951-5955. doi: 10.1073/pnas.89.13.5951.

Puckett, A. M. et al. (2017) 'Measuring the effects of attention to individual fingertips in somatosensory cortex using ultra-high field (7T) fMRI', NeuroImage. Academic Press Inc., 161, pp. 179-187. doi: 10.1016/j.neuroimage.2017.08.014.

Rosen, B. R. and Savoy, R. L. (2012) 'FMRI at 20: Has it changed the world?', NeuroImage. Academic Press, pp. 1316-1324. doi: 10.1016/j.neuroimage.2012.03.004.

Rua, C. et al. (2020) 'Multi-centre, multi-vendor reproducibility of 7T QSM and R2* in the human brain: Results from the UK7T study', NeuroImage. Academic Press Inc., 223, p. 117358. doi: 10.1016/j.neuroimage.2020.117358.

Sanchez-Panchuelo, R. M. et al. (2010) 'Mapping human somatosensory cortex in individual subjects with 7T functional MRI', Journal of Neurophysiology. American Physiological Society Bethesda, MD, 103(5), pp. 2544-2556. doi: 10.1152/jn.01017.2009.

Smith, S. M. et al. (2004) 'Advances in functional and structural MR image analysis and implementation as FSL', in NeuroImage. doi: 10.1016/j.neuroimage.2004.07.051.

Triantafyllou, C. et al. (2005) 'Comparison of physiological noise at 1.5 T, $3 \mathrm{~T}$ and $7 \mathrm{~T}$ and optimization of fMRI acquisition parameters', NeuroImage. Academic Press Inc., 26(1), pp. 243-250. doi: 10.1016/j.neuroimage.2005.01.007.

U. S. Food \& Drug Administration (2017) FDA clears first 7T magnetic resonance imaging device, https://www.fda.gov/news-events/press-announcements/fda-clears-first-7t-magneticresonance-imaging-device. Available at: https://www.fda.gov/news-events/press-

announcements/fda-clears-first-7t-magnetic-resonance-imaging-device (Accessed: 24 March 2021).

Voelker, M. N. et al. (2016) 'The traveling heads: multicenter brain imaging at 7 Tesla', Magnetic Resonance Materials in Physics, Biology and Medicine. Springer Berlin Heidelberg, 29(3), pp. 399-415. doi: 10.1007/s10334-016-0541-8.

Van der Zwaag, W. et al. (2013) 'Digit somatotopy in the human cerebellum: A 7T fMRI study', NeuroImage. Academic Press, 67, pp. 354-362. doi:

10.1016/j.neuroimage.2012.11.041. 